\title{
Metastasis of nasopharyngeal carcinoma: What we know and do not know
}

\author{
Ling-Ling Guo ${ }^{1, a}$, Hai-Yun Wang ${ }^{2,3, a}$, Li-Sheng Zheng ${ }^{1}$, Ming-Dian Wang ${ }^{1}$, Yun Cao ${ }^{4}$, Yang Li $^{5,6}$, \\ Zhi-Jie Liu ${ }^{1}$, Li-Xia Peng ${ }^{1}$, Bi-Jun Huang ${ }^{1}$, Jian-Yong Shao ${ }^{3}$, and Chao-Nan Qian ${ }^{1,7, *}$ \\ ${ }^{1}$ State Key Laboratory of Oncology in South China, Collaborative Innovation Center for Cancer Medicine, Sun Yat-Sen University \\ Cancer Center, Guangzhou 510060, PR China \\ 2 Guangzhou Institute of Pediatrics, Guangzhou Women and Children's Medical Center, Guangzhou 510623, PR China \\ 3 Department of Molecular Diagnostics, Sun Yat-Sen University Cancer Center, Guangzhou 510060, PR China. \\ ${ }_{5}^{4}$ Department of Pathology, Sun Yat-Sen University Cancer Center, Guangzhou 510060, PR China \\ 5 Department of Pathology, the First Affiliated Hospital, Sun Yat-Sen University, Guangzhou 510080, PR China \\ 6 Department of Pathology, Guangzhou Concord Cancer Center, Guangzhou 510555, PR China \\ 7 Department of Radiation Oncology, Guangzhou Concord Cancer Center, Guangzhou 510555, PR China
}

Received 12 January 2021, Accepted 6 July 2021, Published online 9 August 2021

\begin{abstract}
Nasopharyngeal carcinoma (NPC) has the highest metastatic rate among head and neck cancers, with its underlying mechanism not yet fully unveiled. High- versus low-metastasis, NPC cell lines have been established. The footpad-popliteal lymph node metastasis model and other in vivo models have been stably used to study NPC metastasis. The histological appearance and the expression of epithelial-to-mesenchymal transition (EMT) markers might be helpful in selecting high-risk NPC patients for developing post-treatment metastasis. Tested EMT markers and their protein expression levels that correlate with patient disease-free survival in large patient cohorts include E-cadherin, N-cadherin, CD44, Twist, Snail, and Cyclin D1. Epstein-Barr virus (EBV) infection can trigger NPC metastasis from multiple angles via multiple signaling pathways. High endothelial venules are commonly seen in NPC tissues, with their role in NPC metastasis requiring clarification. The molecules that promote and inhibit NPC metastasis are introduced, with a focus on cytokines SPINK6, serglycin, interleukin 8 (IL8), Wnt family member 5A (WNT5A), and chemokine C-C motif ligand 2 (CCL2). Two videos showing NPC cells with and without SPINK6 knocked down are presented. Future directions for studying NPC metastasis are also discussed.
\end{abstract}

Key words: Nasopharyngeal carcinoma, Metastasis promoter, Metastasis inhibitor, High endothelial venule, Pre-metastatic niche, Epithelial-to-mesenchymal transition, EGFR, SPINK6, Video, Epstein-Barr virus, Time-lapse photography.

\section{Background}

The nasopharynx is covered with squamous epithelium, ciliated columnar epithelium, and some transitional regions of these two epithelial types [1]. Submucosal lymphoid tissue in the nasopharynx can be abundant, especially in the posterior superior wall and the roof, known as the adenoid [2]. Adenoid enlargement has been observed in children, and its atrophy is commonly seen in adults.

Nasopharyngeal carcinoma (NPC) is the most common malignancy of the nasopharynx with endemic regions in southern China and Southeast Asia [3, 4]. Although the Epstein-Barr virus (EBV) infection has not yet been conclusively confirmed

*Corresponding author: qianchn@sysucc.org.cn

${ }^{\text {a }}$ First two authors contribute to the study equally. as the etiological factor of NPC tumorigenesis [5], its close relationship with NPC progression has been widely explored and EBV related biomarkers applied in clinical practice to predict NPC occurrence and recurrence [6-8]. Due to the hidden anatomical location of the nasopharynx, early NPC detection has always been a challenge, even in high-incidence areas, with screening for high-risk populations reported to help improve the early detection rate [9]. Consequently, most NPCs in endemic areas are diagnosed at a loco-regionally late stage, with about $10 \%$ of NPC patients suffer from distant metastasis at the time of diagnosis [10]. Although primary NPC is sensitive to radiotherapy and chemotherapy, $15-18.5 \%$ of new NPC patients without distant metastasis will eventually fail from locoregional treatment caused by post-treatment metastasis of the tumor cells [11-13]. Metastasis is the main reason for treatment failure in NPC patients. This is a common challenge in our 
efforts to combat many cancer types as metastasis is the primary cause of cancer-related deaths [14].

Clinically, NPC's most common metastasis pattern is found at two organs/sites [15]. Among the five organs that are frequently colonized by cancer cells, the bone is the most common metastatic NPC site, followed by the lung, the liver, distant lymph nodes, and the brain [15]. Brain metastasis is extremely rare in NPC patients in endemic regions, with the mechanism still unclear [16]. According to the "seed and soil" theory [17], the interaction between NPC cells and the brain microenvironment, some critical features hamper the colonization and/or growth of NPC cells in brain tissues.

While anti-metastasis drugs are not available for many malignancies in current clinical practice, tremendous efforts have been spent over the last two decades trying to unveil the regulatory mechanisms of cancer cells' metastatic ability. Accumulating evidence, including that from NPC studies, shows that this malignant behavior of cancer cells is regulated by hundreds of gene products and multiple signaling cascades [18-22] and the interaction between the tumor microenvironment and tumor cells $[23,24]$. This review article aims to introduce what we know about NPC metastasis from different angles and discuss the future of NPC research.

\section{Cellular and animal models for studying NPC metastasis}

It is critical to establish cellular and animal models for NPC metastasis. Our efforts [25] and others' [26] have provided two stable high-metastasis cellular clones, S18 and 5-8F, from two different NPC parental cell lines CNE2 and SUNE1, respectively. Two low-metastasis clones, S22 and S26, have also been established by our team [25]. These cellular clones have been widely used to explore the molecular mechanisms regulating NPC metastasis [27-37].

Metastasis is defined by the spread of cancer cells from one organ to other organs; therefore only in vivo models can be used to demonstrate this phenomenon convincingly. There are two major categories of murine models for studying metastasis [38]. One is the spontaneous model, where a primary tumor is generated, and metastatic lesions occur later in other organs. Two is the experimental metastasis model, where cancer cells are injected directly into the bloodstream to spread to distant organs without the formation of a primary tumor. In NPC studies, the widely used experimental metastasis model is the tail-vein injection of cancer cells into nude mice to generate pulmonary lesions. It is believed that the spontaneous metastasis model mimics the whole process of metastasis including the detachment of cells from the primary tumor, the invasion of cells into the surrounding normal tissues, intravasation, the survival of cancer cells in the bloodstream, extravasation, the colonization of cells in a recipient organ, the formation of micrometastasis, angiogenesis in the metastatic lesion, and the formation of macrometastasis. In contrast, the experimental metastasis model skips several initial steps of metastasis by releasing the cancer cells directly into the bloodstream.

The ideal spontaneous metastasis model generates a primary tumor in a respective organ, for example, via orthotopic injection of breast cancer cells into a breast fat pad of a mouse to grow a primary tumor before pulmonary metastasis. However, there is no reliable orthotopic NPC mouse model for studying NPC metastasis. A convenient alternative is the spontaneous metastasis model for NPC study using a footpad injection before popliteal lymph node metastasis in a nude mouse $[25,39]$. In such a model, NPC cells are subcutaneously injected into a hind footpad of a nude mouse to generate a primary tumor; metastasis will then occur in the sentinel lymph node (which is a popliteal lymph node in this model) two to four weeks after the initial inoculation of the cancer cells. Another widely used spontaneous metastasis model is via intrasplenic injection of NPC cells to generate liver metastasis [37].

\section{Pathological morphology and metastatic propensity}

According to the World Health Organization's (WHO) pathological classification, NPCs are divided into three types: keratinizing squamous cell carcinoma, non-keratinizing differentiated carcinoma, and non-keratinizing undifferentiated carcinoma [40]. However, this classification does not provide sufficient information to predict patient outcomes, especially the occurrence of post-treatment metastasis [41, 42]. A multicenter study proposed a new NPC pathological classification using a training cohort of 2716 patients, a retrospective validation cohort of 1702 patients, and a prospective validation cohort of 1613 patients [42]. With a total of 6031 patients, it is the most comprehensive study to date on NPC pathological classification. There are four types of NPC in this new classification based on morphologic features: epithelial carcinoma (EC), sarcomatoid carcinoma (SC), mixed sarcomatoid-epithelial carcinoma (MSEC), and squamous cell carcinoma (SCC) (Figure 1). Importantly, patients with sarcomatoid carcinoma have a relatively poorer overall survival rate [42]. Given that metastasis is the main reason for treatment failure in NPC, the metastatic ability of sarcomatoid NPC cells cannot be neglected. Interestingly, in our previously published NPC cohort [43], sarcomatoid NPC had a significant EMT status compared with other histological NPC types (Figure 2). Further studies to clarify the metastatic potential of sarcomatoid NPC are warranted for future selection of high-risk patients for distant metastasis, with this group of patients believed to benefit from anti-metastasis treatments.

\section{Tumor microenvironment and pre-metastatic niche for NPC metastasis}

\section{High endothelial venules (HEVs) in NPC tissues and pre-metastatic regional lymph nodes}

HEVs are unique blood vessels that facilitate the extravasation of lymphocytes from the bloodstream to lymphoid tissues (not including the spleen) [44]. HEVs are mainly localized in lymphoid organs, except for the spleen. HEVs express a specific mixture of glycoproteins, named peripheral lymph node addressin (PNAd), which are recognized by the membrane homing receptor L-selectin on the lymphocytes to fulfill their 

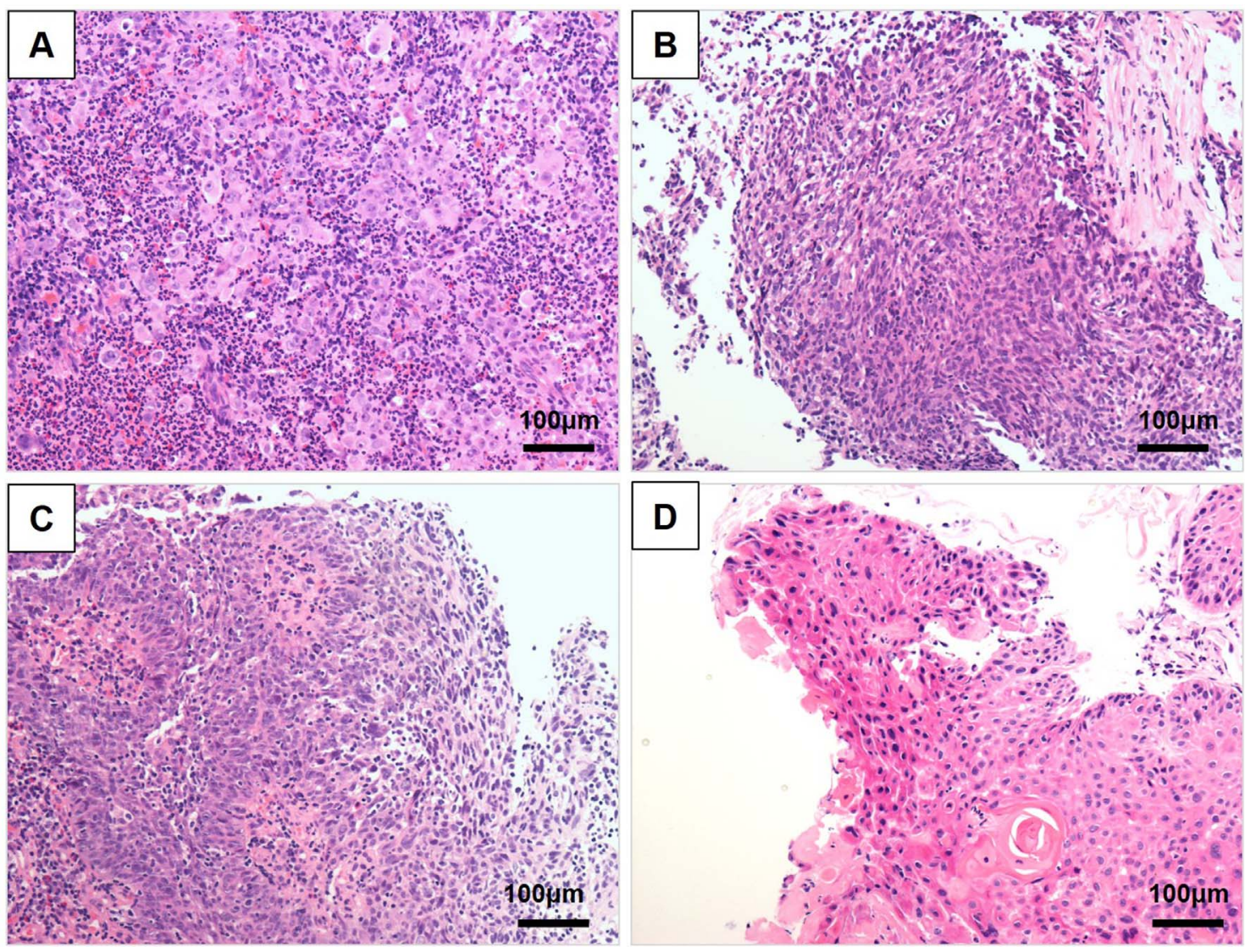

Figure 1. Representative morphologic character of tumor cells according to the new NPC classification. Typical NPC histological appearances. The epithelial carcinoma (EC) subtype shows round cells with prominent nucleoli and a pavement-like appearance, with a low nucleus: cytoplasm ratio (A). The sarcomatoid carcinoma (SC) subtype features irregular small cells and uniform medium-sized spindle cells (B). The mixed sarcomatoid-epithelial carcinoma (MSEC) subtype is characterized by a scattered infiltration of large, round cells in the spindle cell carcinomatous tissue without obvious boundaries (C). The squamous cell carcinoma (SCC) subtype shows well-differentiated keratinizing SCC with a large number of whorls and keratin (D).

immune function. However, our previous study has found that the primary tumor can remodel HEVs in the sentinel lymph node that drain the primary tumor before the arrival of NPC cells into vessels with an enlarged lumen, thinner wall, and the ability to transport large amounts of red blood cells, indicating a functional shift of HEVs from immune function to oxygen supply provider [25, 45]. After the arrival of the metastatic cancer cells, the metastatic colony further highjacks the functionally shifted HEVs to become part of the tumor vasculature with subsequent remodeling evident by partial to complete loss of PNAd expression; this procedure is termed vessel co-option [46]. Following the engagement of tumor cells with HEVs, tumor cells can enter the bloodstream more efficiently, thereby generating distant metastasis [47].

Notably, the nasopharynx is a lymphoid organ with HEVs present in the nasopharyngeal mucosa (Figure 3). NPC cells are commonly close to the HEVs or even surrounding the HEVs. Cellular clusters inside the HEVs of NPC tissues are regularly observed, and these clusters can be made up of cancerous cells. The role of HEVs in NPC metastasis warrants further clarification.

\section{Myeloid-derived suppressor cells}

Many non-cancerous cell types have been reported to form a pre-metastatic niche in lung cancer, including bone marrowderived cells, myeloid-derived suppressor cells (MDSCs), myeloid cells, monocytes, granulocytes, neutrophils, macrophages, regulatory $\mathrm{T}$ cells, endothelial progenitor cells, and hematopoietic progenitor cells [16]. Targeting the pre-metastatic niche formed by MDSCs has been suggested as a potential anticancer treatment strategy [48].

The important roles of MDSCs in NPC have been revealed. MDSC are a heterogeneous population of immune cells from the myeloid lineage that expand during infection, inflammation, and cancer progression [49]. A preliminary study found that MDSCs can promote NPC metastasis through over-expression of cyclooxygenase-2 (COX-2) in the cancer cells and subsequently activate beta-catenin/Transcription factor 4 (TCF4) signaling [50].

More efforts are needed to elucidate the cross-talk among multiple cell types underlying the formation of NPC pre-metastatic niches. 


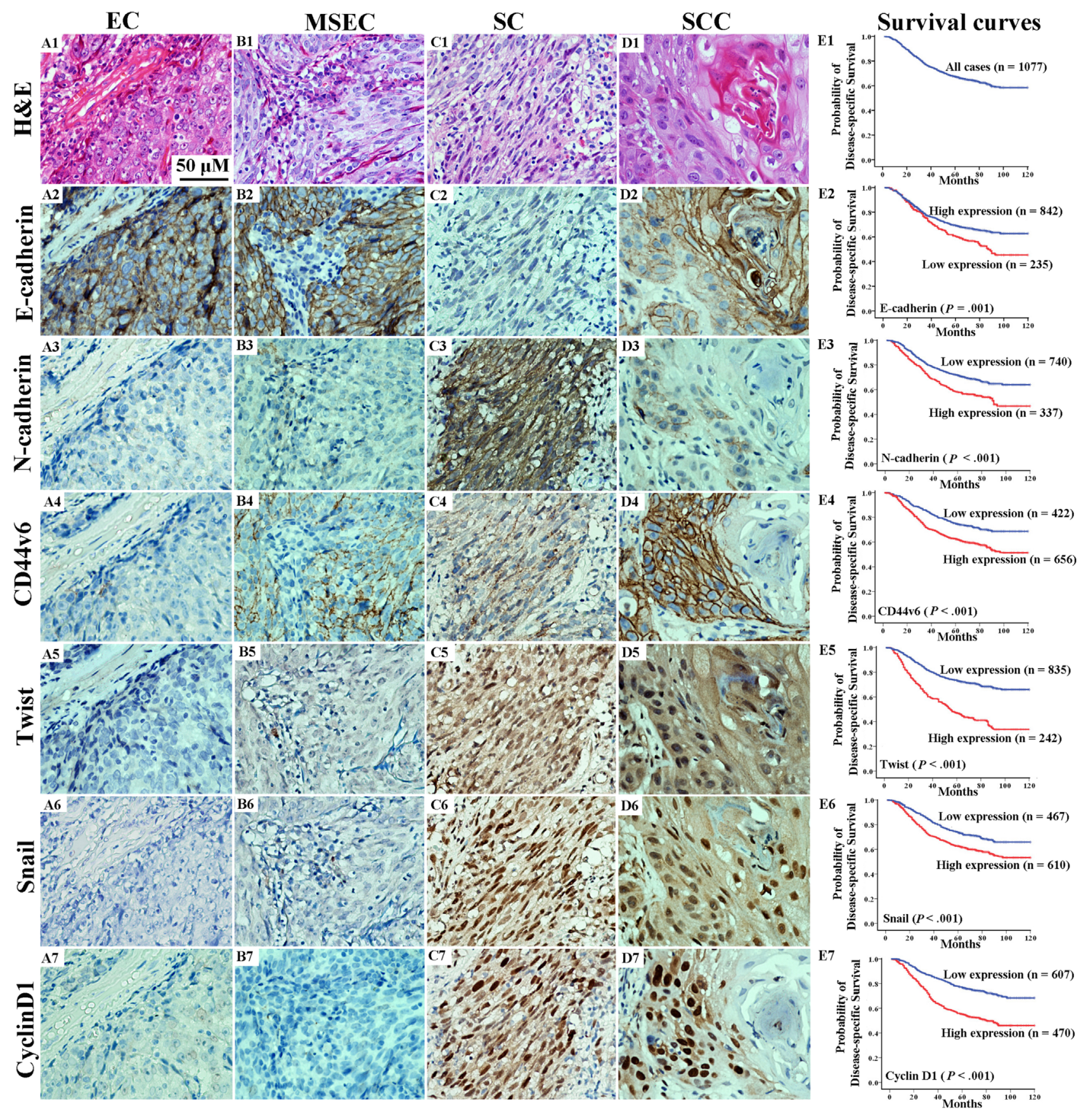

Figure 2. The expressions of epithelial-mesenchymal transition (EMT) markers in NPC and their correlations with patient disease-free survivals. The hematoxylin and eosin (H\&E) staining features of EC, MSEC, SC, and SCC subtypes (line 1, A1, B1, C1, and D1). Preserved E-cadherin expression in EC (A2), MSEC, (B2), and SCC (D2) in contrast with loss of expression in SC (C2) are shown in line 2. N-cadherin IHC staining with overexpression in SC (C3) and absent expression in EC (A3), MSEC (B3), and SCC (D3) are shown in line 3. CD44v6 IHC staining with a lack of expression in EC (A4) and expression in MSEC (B4), SC (C4), and SCC (D4) is shown in line 4. Twist IHC staining showing no expression in EC (A5) and MSEC (B5) and overexpression in SC (C5) and SCC (D5) (line 5). Snail IHC staining showing no expression in EC (A6) and MSEC (B6) and overexpression in SC (C6) and SCC (D6) (line 6). Cyclin D1 IHC staining with no expression in EC (A7) and MSEC (B7) and overexpression in SC (C7) and SCC (D7) (line 7). All histological images were taken using the same magnification shown in A1. Panel E1 shows the disease-free survival curve of all 1077 NPC patients. Panel E2-7 shows the survival curve of 1077 patients with IHC staining of EMT markers. The 5-year disease-free survival rates of NPC patients with low- vs. high-level expression. The indicated markers are: $60.8 \%$ vs. $69.4 \%$ for E-cadherin (E2), $71.9 \%$ vs. $58.3 \%$ for N-cadherin (E3), $75.1 \%$ vs. $62.7 \%$ for CD44v6 (E4), $73.5 \%$ vs. $47.1 \%$ for Twist (E5), $74.0 \%$ vs. $62.7 \%$ for Snail (E6), and $77.2 \%$ vs. $55.3 \%$ for cyclin D1 (E7). The $P$-values for all comparisons are less 0.01 . The largest difference in 5-year disease-free survival was in the Twist expression comparison, with a difference of $26.5 \%$. 


\section{Epithelial to mesenchymal transition in NPC metastasis}

The transition of epithelial cells to mesenchymal like-cells is called epithelial-to-mesenchymal transition (EMT). It is widely believed that EMT is the initial step in the cascade of cancer metastasis $[51,52]$. Although, some controversial findings suggest that EMT is dispensable for metastasis [53]. In addition to the morphological alteration of the cells, EMT is more commonly determined by overexpression of mesenchymal markers and a loss of epithelial markers. Over a dozen EMT markers have been widely used in cancer research [54]. Among them, according to our large-cohort study findings, the elevated expressions of E-cadherin are correlated with better NPC patient prognosis. In contrast, the elevated expressions of N-cadherin, CD44v6, Twist, Snail, and Cyclin D1 are correlated with poorer NPC patient prognosis (Figure 2).

Based on our findings and other reports, the metastatic ability of NPC cells correlates with EMT [31, 37, 55, 56]. Suppressing EMT by CLCA2 and NOTCH2 can inhibit NPC metastasis in animal models [35, 57]. However, direct evidence of NPC metastasis inhibition by reversing EMT or inducing mesenchymal-to-epithelial transition is unconvincing, which is part of the much larger dilemma in cancer biology studies [58]. Moreover, some pro-metastasis cytokines do not induce typical EMT when they promote NPC metastasis. Serglycin, for example, can strongly promote NPC metastasis but only increases vimentin expression among various detected EMT markers [39]. These findings suggest that the metastatic ability of NPC cells could be both EMT-dependent and EMTindependent.

\section{Epstein-Barr virus (EBV) infection and NPC metastasis}

EBV is a latent infection in more than $90 \%$ of adults worldwide [59]. It is associated with NPC and lymphoid malignancies, such as Burkitt's lymphoma [60]. Almost all NPCs in endemic regions are associated with EBV infection [61]. The expression of the EBV genome in NPC cells comes from protein-coding and non-protein-coding genes. The former includes EBV latent membrane proteins (LMPs) and EBV nuclear antigens (EBNAs). The latter includes EBV-encoded small non-polyadenylated RNAs (EBERs) and microRNAs4 [62]. Two small EBV encoded RNAs, EBER1 and EBER2, are the most abundant viral transcripts in latent EBV-infected NPC cells [63]. The EBV miRNAs are mainly BamHI-A region rightward transcripts (BARTs) and BamHI fragment $\mathrm{H}$ rightward open reading frame 1 (BHRF1) miRNA clusters $[64,65]$. In the latent $1-2$ types, miRNAs encoded by BART are detectable, whereas miRNAs from BHRF1 are mainly detectable in latent type 3 and lytic infected cells [66]. EBV genes have been reported to be involved in NPC malignant progression, including EMT, cellular motility, angiogenesis [67], metastasis [68], and treatment resistance [66].

Target cells of EBV mainly include B lymphocytes and epithelial cells. EBV glycoproteins $\mathrm{gHgL}$, and $\mathrm{gB}$, are regarded as the core fusion machinery for membrane fusion of EBV with
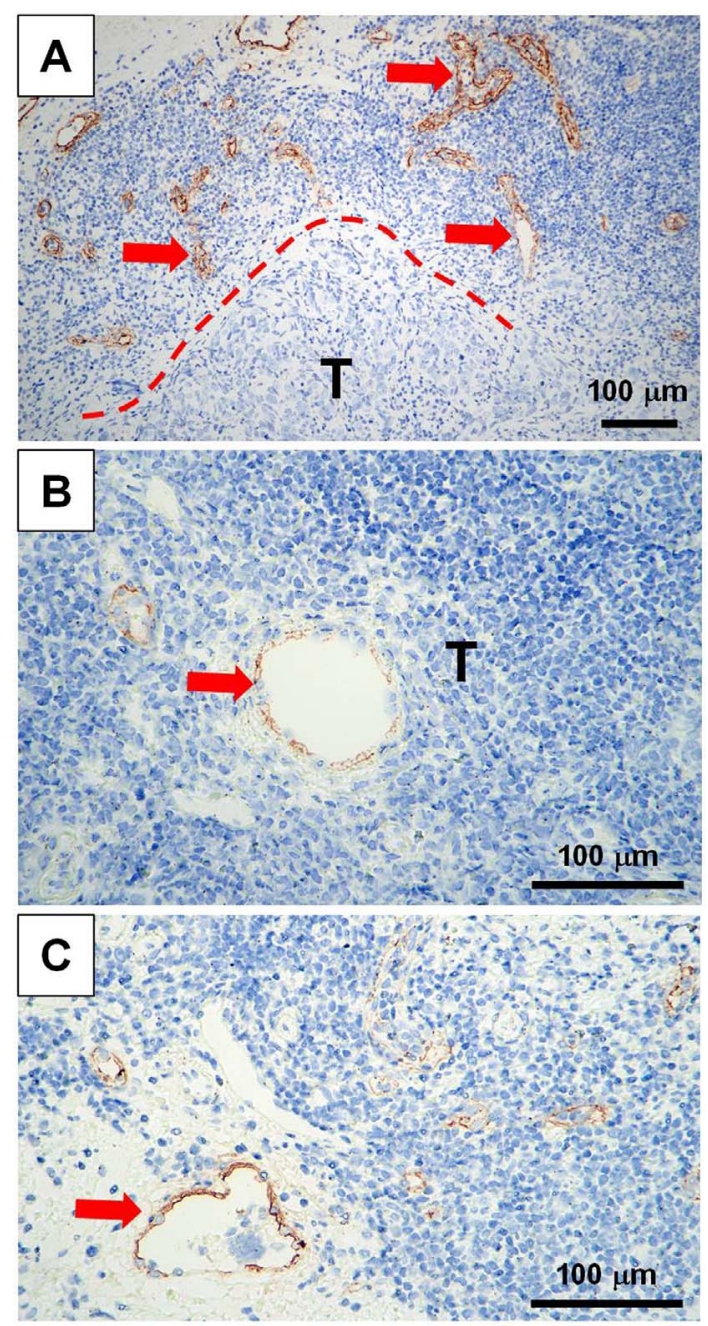

Figure 3. High endothelial venules in NPC tissues. High endothelial venules (HEVs) stained by MECA-79 antibody to visualize peripheral node addressin (PNAd). (A) The HEVs are indicated by arrows located in the periphery of the tumor nest (T). (B) One HEV is surrounded by tumor cells. (C) A NPC tissue section with an embolus cell cluster within a HEV (arrow).

all cell types [69]. EBV gB interacts with neuropilin 1, which may activate neuropilin 1-dependent EGFR (RTKS) signaling and promote EBV to enter nasopharyngeal epithelial cells through macropinocytosis and lipid raft-dependent endocytosis [70]. F-box only protein 2 (FBXO2) directly binds to $\mathrm{gB}$ $\mathrm{N}$-glycosylation sites promoting $\mathrm{gB}$ degradation via the ubiquitin-proteasome pathway. A recent study found that depletion of FBXO2 promotes gB localization on the surface of nasopharyngeal epithelial cells, resulting in enhanced membrane fusion and viral entry [71].

Two small EBV encoded RNAs, EBER1 and EBER2, are the most abundant viral transcripts in latent EBV-infected NPC cells [63]. The overexpression of EBERs can trigger the secretion of pro-inflammatory cytokines, macrophage colonystimulating factor (M-CSF), and monocyte chemo-attractant protein (MCP-1) via nuclear factor- $\kappa \mathrm{B}(\mathrm{NF}-\kappa \mathrm{B})$ and IRF3 signaling pathways to form a pro-tumorigenic inflammatory 

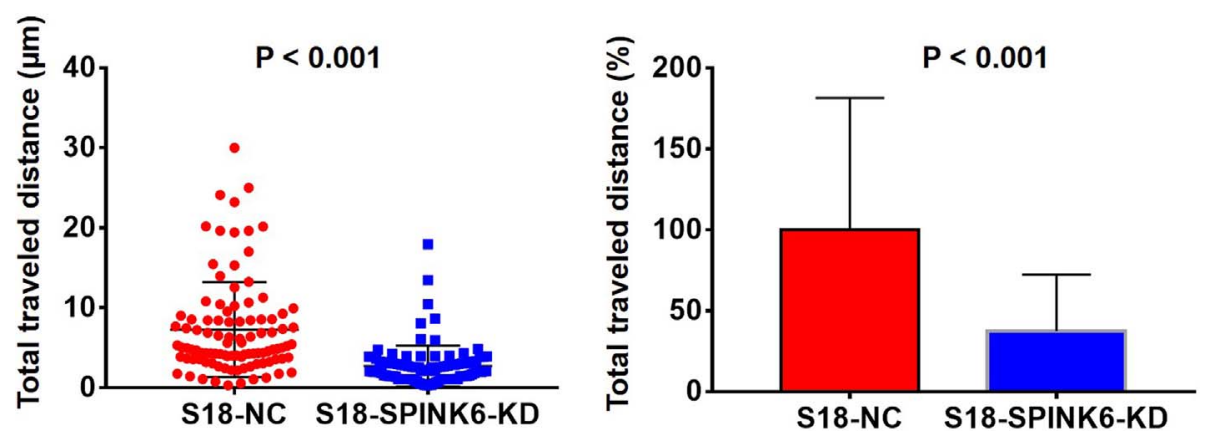

Figure 4. Migratory ability of NPC S18 cells is suppressed by knocking down SPINK6 expression. S18-NC and S18-SPINK6-KD cells (GFPtagged) were seeded in a 96-well plate (2000 cells per well) and cultured in DMEM with $10 \% \mathrm{FBS}$ in $5 \% \mathrm{CO}_{2}$ at $37{ }^{\circ} \mathrm{C}$ for $24 \mathrm{~h}$. Next, the plate was placed under a Nikon ECLIPSE Ti2 Inverted Microscope System supplied with $5 \% \mathrm{CO}_{2}$ at $37{ }^{\circ} \mathrm{C}$. Images of random fields at $100 \times$ magnification were taken automatically every $30 \mathrm{~min}$ for $72 \mathrm{~h}$. A total of 145 sequential images of each field were taken, generating a video to display the movement of the cells at a speed of 15 FPS (frame/sec) (see Videos 1 and 2). The locations and moving tracks of the cells were analyzed using Image $\mathbf{J}$ software and the distance of movement between sequential images every 30 min were calculated. The videos displaying the movement of S18-NC and S18-SPINK6-KD cells are shown in Videos 1 and 2. The accumulated distance traveled in $72 \mathrm{~h}$ of 100 randomly selected cells from each cell line were calculated and are shown in A and B.

tumor microenvironment, which consequently promotes NPC development [72].

In an analysis of genome-wide profiling of EBV integration, the EBV genome can integrate into the human genome resulting in down-regulation of the genes coding tumor necrosis factor-alpha-induced protein 3 (TNFAIP3), Parkinson disease2 (PARK2), and cyclin-dependent kinase 15 (CDK15) [73]. Recent findings revealed that all of these three gene products are either motility or metastatic inhibitors. TNFAIP3 inhibits the migration and invasion of NPC cells [74], PARK2 suppresses the metastasis of glioblastoma cells [75], and CDK15 is a metastatic breast cancer inhibitor [76].

The latent membrane protein 1 (LMP1) is an EBV-encoded protein and regulates biological abnormalities of NPC through various signaling pathways [69], including cell migration (SOCE, integrin-alpha5 and N-cadherin) [77, 78] angiogenesis and permeabilization (SOCE) [79], necroptosis (RIPK1/3) [80], stemness (PI3K/AKT) [81], and metastasis [82]. A recent highthroughput sequencing study proved that LMP1 constitutively activated NF- $\kappa B$ signaling [83], with LMP1-activated NF- $\kappa B$ binding to the promoter region of the microRNA-203 gene downregulating miR-203, leading to EMT and potentially to metastasis propensity [84].

The transcriptional factor Twist, a highly conserved basic helix-loop-helix protein, is essential for early embryogenesis, which promotes EMT and plays an essential role in metastasis. LMP1 can induce Twist via the NF- $\kappa \mathrm{B}$ pathway activating the EMT program and promoting metastasis [85].

LMP2A is another membrane protein encoded by the EBVLMP2 gene. It is consistently expressed in over $98 \%$ of NPCs [86] and activates PI3-K/Akt, JNK/SAPK, ERK-MAPK, and Wnt/beta-catenin signaling to regulate cell growth, apoptosis, and differentiation $[67,86]$, as well as competitively binding to Syk to suppress the interaction between SYK and ITGbeta4 to promote NPC cellular migration and invasion [87].

The EBV-encoded microRNAs that can activate invasion and metastasis of NPC cells include miR-BART6-3p,
miR-BART7-3p, miR-BART8-3p, miR-BART9, miR-BART13, and miR-18-5p [88].

Taken together, the EBV infection is an important factor in promoting NPC cellular motility and metastasis from multiple angles and involves multiple signaling pathways.

\section{Metastatic promoters in NPC}

\section{Cytokines and their receptors}

Multiple cytokines are NPC metastasis promoters. These secreted molecules commonly act through both autocrine and paracrine manners in promoting NPC metastasis.

Kazal-type serine proteinase inhibitor 6 (SPINK6) possesses the normal physiological function of inhibiting several kallikrein-related peptidases (KLKs), specifically, KLK5, KLK7, and KLK14 [89]. Our group revealed that SPINK6 is also the ligand of epidermal growth factor receptor (EGFR) in NPC cells which induces the dimerization of EGFR and results in activating downstream AKT signaling for metastasis, and this function of SPINK6 is independent of its serine protease-inhibitory activity [37]. Using time-lapse photography, the total traveled distance of NPC S18 cells with our without SPINK6 knockdown is quantified in Figure 4 and shown in Videos 1 and 2.

Serglycin is a proteoglycan with its core peptide coded by the SRGN gene in humans. It is a family member of small proteoglycans with serine-glycine dipeptide repeats and modified with different types of glycosaminoglycan side chains [90]. In highmetastatic NPC cells, we found that serglycin promotes NPC metastasis through binding to its receptor CD44 and consequently activates MAPK/ $\beta$-catenin signaling [39, 91]. More interestingly, serglycin can also upregulate CD44 expression in an autocrine manner to maintain self-renewal of NPC cells [91].

As a member of the CXC chemokine family, interleukin 8 (IL8), alternatively known as CXCL8, is secreted by endothelial cells, neutrophils, tumor-associated macrophages, 


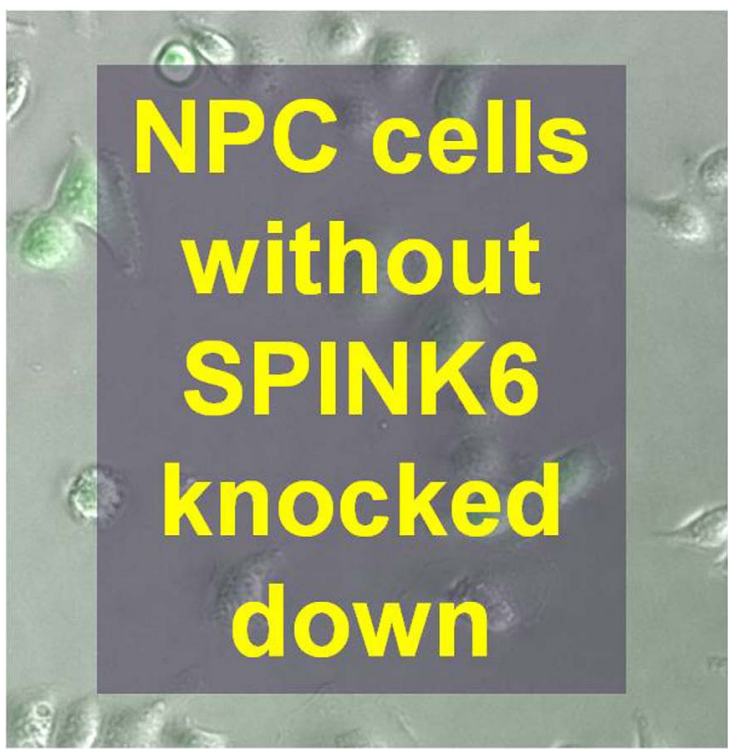

Video 1. Migratory ability of NPC S18 cells transfected with the scrambled control shRNA (S18-NC). The time-lapse photography images described in Figure 4 of the NPC S18 cells without SPINK6 knocked-down (S18-NC). Notably, the cells are more active in comparison with Video 2. https://vcm.edpsciences.org/10.1051/vcm/ 2021003\#V1

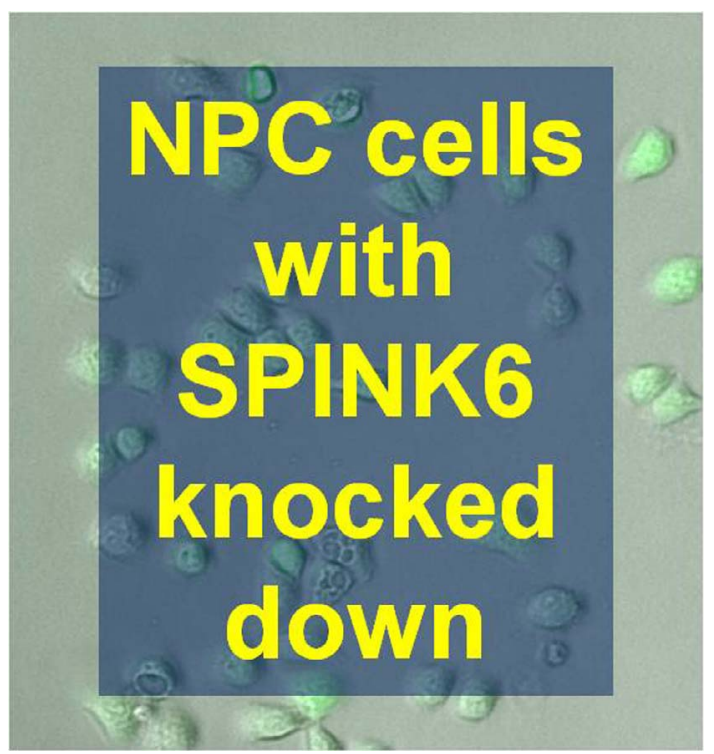

Video 2. Migratory ability of NPC S18 cells transfected with shRNA against SPINK6 (S18-SPINK6-KD). The time-lapse photography images described in Figure 4 of the NPC S18 cells with SPINK6 knocked-down (S18-SPINK6-KD). Notably, the cells are less active in comparison with Video 1. https://vcm.edpsciences.org/10.1051/ $\mathrm{vcm} / 2021003 \# \mathrm{~V} 2$

and cancer cells [92]. IL8 binds to two cellular membrane receptors CXCR1 and CXCR2, for its pro-inflammatory functions. We previously reported that high-metastatic NPC cells could secrete IL8 promoting NPC metastasis, with AKT signaling being the downstream activated pathway [31]. E-cadherin has been regarded as an inhibitor of NPC metastasis [93, 94]. Another study of ours revealed that IL8 suppresses E-cadherin expression in NPC cells by enhancing E-cadherin promoter DNA methylation [95].

Wnt family member 5A (WNT5A) belongs to the large WNT family of cysteine-rich secreted glycoproteins and binds to Frizzled family receptors (Fzd) and low-density lipoprotein receptor-related protein 5/6 co-receptors (LRP5/6) [96]. We reported WNT5A promotes EMT in NPC cells, induces the accumulation of $\mathrm{CD} 24^{-} / \mathrm{CD} 44^{+}$stem-like cells, and promotes NPC metastasis via protein kinase C (PKC) signaling [97].

Chemokine $\mathrm{C}-\mathrm{C}$ motif ligand 2 (CCL2) is a cytokine that can bind to its receptor chemokine $\mathrm{C}-\mathrm{C}$ motif receptor type 2 (CCR2) and trigger ERK1/2-MMP2/9 signaling for promoting NPC metastasis [98].

Other cytokines reported to promote NPC cellular motility in vitro, and hence are potential NPC metastasis-promoters, include TNFAIP2 [99], IL-6 [100], and IL-17A [101].

\section{Cell-surface proteins as metastasis drivers}

Epidermal growth factor receptor (EGFR) belongs to the ErbB receptor tyrosine kinase (TK) family, with its dysregulation found in multiple human cancers. The activation of EGFR by its native ligand EGF for deteriorating NPC metastasis has recently been confirmed [102], with PKM2 one of its key downstream molecules. SPINK6 is another ligand that can bind to EGFR and promote NPC metastasis [37].

Urokinase-type plasminogen activator receptor (UPAR) is a glycosyl phosphatidylinositol-anchored membrane protein possessing multiple functions, which its ligand uPA can activate through the binding of vitronectin [103]. We reported that uPAR promotes NPC cell growth and metastasis via activating the JAK-STAT pathway [27].

Ezrin is a member of the Ezrin-Radixin-Moesin protein family functioning as a linker between the plasma membrane and actin cytoskeleton, which is also known as a metastatic promoter in breast cancer [104]. Suppression of Ezrin expression results in an inhibition of NPC cellular migration and invasion [105], and its mRNA stability can be enhanced by circular RNA circARHGAP12 [106].

Glypican 6 is a putative cell surface co-receptor for growth factors. Its over-expression promotes NPC cellular motility, which might promote metastasis but is yet to be confirmed [107]. Another membrane protein, TSPAN8, has also been reported to promote NPC cellular motility via activating Akt/MAPK signaling [32].

\section{Intracellular proteins as metastasis drivers}

Two important intracellular proteins that activate the PI3K/AKT pathway and subsequently stimulate NPC metastasis are the non-receptor tyrosine kinase c-Src [108] and $\mathrm{Ca}^{+}$-dependent phospholipid-binding protein annexin A1 [109]. Another intracellular protein that can also trigger AKT signaling for enhancing NPC cellular motility is FLJ10540 [110] and therefore possesses the potential to promote NPC metastasis. FLJ10540 
Cdc42-interacting protein 4 (CIP4) is encoded by the TRIP10 gene. Our group found that CIP4 is required for NPC cellular motility and metastasis. CIP4 promotes the activation of N-WASP that controls invadopodia formation. Meanwhile, CIP4 activates EGFR signaling resulting in an up-regulation of matrix metalloproteinase 2 (MMP2) [34].

Serine beta-lactamase-like protein (LACTB) is an enzyme encoded by the $L A C T B$ gene and located in the mitochondrial intermembrane space. LACTB's normal function is to regulate lipid metabolism. Our recent findings show that LACTB promotes NPC metastasis by activating ERBB3/EGFR-ERK signaling [111].

Zinc finger protein SNAI1 (SNAIL) is a transcriptional repressor that downregulates the expression of E-cadherin. The over-expression of SNAIL can promote NPC metastasis partially by down-regulating telomere length regulation protein TEL2 [112].

\section{Non-coding RNAs}

Accumulating evidence shows the important roles of noncoding RNAs in promoting NPC metastasis. Long non-coding RNA AFAP1-AS1 competes with endogenous RNA miR-423$5 \mathrm{p}$ to promote NPC metastasis through activating Rho/Rac signaling [113]. Long non-coding RNA FAM225A acts as ceRNA to sponge miR-590-3p/miR-1275 and upregulates ITGB3 to promote NPC metastasis [114]. Long non-coding RNA LINC01503 stimulates NPC metastasis by recruiting splicing factor proline-and glutamine-rich (SFPQ) to activate Fos-like 1 (FOSL1) transcription [115]. MicroRNA-494-3p promotes NPC cellular motility and growth by targeting Sox 7 [116]. It is expected that more and more non-coding RNAs regulating NPC metastasis will be identified.

\section{Metastatic inhibitors in NPC}

In recent years, several NPC metastatic inhibitors have been revealed acting through a variety of mechanisms illustrating the complexity of NPC metastasis.

\section{Transcriptional regulation}

TEL2 belongs to E26-transformation specific transcription factors, which inhibit NPC metastasis by suppressing the expression of serpin family E member 1 (SERPINE1) [36].

Transcription factor zinc finger protein 582 (ZNF582) inhibits NPC metastasis via regulating the transcription and expression of adhesion molecules nectin-3 and neurexin 3 (NRXN3) [117].

\section{Membrane receptor regulation}

Toll-like receptor 3 (TLR3) activation inhibits NPC metastasis by down-regulating the expression of chemokine receptor CXCR4 [118].

\section{Central pathway regulation}

Shroom family member 2 (SHROOM2) inhibits NPC metastasis via RhoA-ROCK signaling-dependent and -independent mechanisms [119].
Depending on integrin $\beta 1$ and merlin interaction, CHL1 suppresses NPC metastasis by inhibiting the PI3K/ AKT signaling pathway [120].

Nidogen-2 (NID2) suppresses NPC metastasis by inhibiting EGFR/Akt and integrin/FAK/PLC $\gamma$ metastasis-related pathways [121].

The critical inhibitory effects of non-coding RNAs against NPC metastasis have also been discovered with the findings that NKILA, a long non-coding RNA, inhibits NPC metastasis via suppressing NF- $\kappa B$ signaling [122], and MiR-99a inhibits NPC metastasis through targeting HOXA1 [123].

\section{Other mechanisms}

Other proteins and non-coding RNAs that can inhibit the migration and invasion of NPC cells and therefore possess the potential to inhibit NPC metastasis in vivo include TNFAIP3 [74], bactericidal/permeability-increasing-fold-containing family B member 1 (BPIFB1) [124], MYH10 [125], miR-30e-5p [126], miRNA-101 [127], and miR-451 [128].

\section{Other oncogenes and tumor suppressor genes in NPC cells}

Studies on chromosomal alterations in NPC cells have revealed the association between allelic losses on the short arms of chromosomes 3 and 9 and inactivation of several tumor suppressor genes, particularly p14, p15, and p16 [129-131]. Other genomic studies have found that HLA genes residing at the $\mathrm{MHC}$ region on chromosome 6p21 and other loci outside the MHC (e.g., TNFRSF19 on 13q12, MECOM on 3q26) are risk loci associated with NPC occurrence and development [10]. Recently, some molecular signatures that can predict NPC's prognosis independent of TNM staging have been identified. For locoregionally advanced nasopharyngeal carcinoma, a gene-expression signature of 13 genes has been generated to predict the risk of survival, the risk of distant metastasis and the effect of concurrent chemotherapy in NPC patients [132]. Consisting of five features (B7-H3TAIC, IDO-1TAIC, VISTATAIC, ICOSTAIC, and LAG3TAIC), the immune checkpointbased signature (ICS) has also been proposed to predict the risk of overall, distant metastasis-free and disease-free in NPC, which was significantly linked to survival in patients with a high EBV-DNA load [133].

In addition to molecular signatures, an integrated molecular analysis identified two NPC-associated oncogenes, lymphotoxin beta receptor (LTBR) and Cyclin D1. LTBR encodes a member of the TNFR family, which activates the main signaling pathways, including NF-кB and c-Jun N-terminal kinase. In a recent study, ectopic expression of LTBR correlated with upregulated NF-кB activity in nasopharyngeal epithelia cells [134].

Cyclin D1 is a cell cycle regulating protein, which regulates the G1 to S-phase transition. It forms complexes with CDK4 or CDK6, subsequently phosphorylates the retinoblastoma tumor suppressor protein, and initiates DNA synthesis. It is overexpressed in more than $90 \%$ of primary NPCs. Array-based comparative genomic hybridization analysis identified Cyclin 
D1 as the target oncogene in the 11q13.3 amplification of NPC [135]. Cyclin D1 transcription is regulated by the constitutively activated NOTCH3 signaling [136]. In vitro experiments have also demonstrated that cellular proliferation and metastasis are dependent on Cyclin D1 activation or other mechanisms for cell cycle inactivation (e.g., p16).

In the majority of NPCs, the PI3K-AKT signaling pathway is constitutively activated. The PIK3CA, located at 3q26.1, encodes the $110-\mathrm{kDa}$ catalytic subunit of phosphatidylinositol 3-kinase (PI3K) and is a common oncogenic alteration in NPC. Phosphatidylinositol 3,4,5-triphosphate (PIP3) is generated as a second messenger when PI $3 \mathrm{~K}$ is coupled with the $85-\mathrm{kDa}$ subunit. The PIP3, in turn, activates AKT signaling and a wide range of downstream targets that promote cell proliferation, inhibit apoptosis, and trigger cellular motility. Copy number gain and amplification of PIK3CA have been found in $75 \%$ and $20 \%$ of primary tumors, respectively, suggesting the critical roles of AKT signaling in NPC progression.

\section{Future directions}

It is evident that our understanding of NPC metastasis is limited, and a multitude of investigations are warranted. It is expected that the characterization of key molecules promoting or inhibiting NPC metastasis might be useful in identifying high-risk patients at the time of diagnosis, those prone to post-treatment distant metastasis, and developing targeted therapeutics for clinical intervention.

To use the well-established NPC cells with different metastatic abilities for anti-metastasis drug development is another plausible direction. Theoretically, these cells can be used to screen any compound library for identifying the lead antimetastasis compounds. Of course, a live cell-based high throughput screening system needs to be established for this purpose, and more effective preclinical models need to be optimized to validate the identified lead compounds.

A better understanding of the pre-metastatic niche will help develop novel approaches to block the further spread of NPC cells from the sentinel or regional lymph nodes to distant organs. By doing this, the development of cancer progression can be significantly slowed down and patient survival meaningfully prolonged.

Taken together, metastasis in NPC is the bottleneck for further improvement of treatment outcomes. We can overcome this challenge by developing an early alert system for NPC metastasis based on our deeper understanding of its underlying mechanisms and developing anti-metastasis agents.

\section{Conflict of interest}

The authors declare that they do not have any conflict of interest.

Acknowledgements. This work was supported by grants from the National Natural Science Foundation of China (No. 82073220, No. 81872384, No. 81672872 and No. 81472386 to C.Q., No. 81773279 to Y.S., No. 81773162 and No. 81572901 to B.H.), the Provincial Natural Science Foundation of Guangdong, China (No. 2016A030311011 to C.Q., and No. 2017A030313866 to
B.H.), and a research program of Sun Yat-sen University (No. 84000-18843409 to C.Q.).

\section{References}

1. Ali MY. Histology of the human nasopharyngeal mucosa. J Anatomy. 1965;99(Pt 3):657-672.

2. Ali MY. Pathogenesis of cysts and crypts in the nasopharynx. J Laryngol Otol. 1965;79:391-402.

3. Cao SM, Simons MJ, Qian CN. The prevalence and prevention of nasopharyngeal carcinoma in China. Chin J Cancer. 2011;30 (2):114-119.

4. Wee JT, Ha TC, Loong SL, et al. Is nasopharyngeal cancer really a "Cantonese cancer"? Chin J Cancer. 2010;29(5):517-526.

5. Gu AD, Zeng MS, Qian CN, The criteria to confirm the role of Epstein-Barr virus in nasopharyngeal carcinoma initiation. Int $\mathrm{J}$ Mol Sci. 2012;13(10):13737-13747.

6. Chan KCA, Woo JKS, King A, et al. Analysis of plasma Epstein-Barr Virus DNA to screen for nasopharyngeal cancer. N Engl J Med. 2017;377(6):513-522.

7. Chen QY, Guo SY, Tang LQ, et al. Combination of tumor volume and Epstein-Barr Virus DNA improved prognostic stratification of stage II nasopharyngeal carcinoma in the intensity modulated radiotherapy era: a large-scale cohort study. Cancer Res Treat. 2018;50(3):861-871.

8. Lin JC, Wang WY, Chen KY, et al. Quantification of plasma Epstein-Barr virus DNA in patients with advanced nasopharyngeal carcinoma. N Engl J Med. 2004;350(24):2461-2470.

9. Lam WKJ, Jiang P, Chan KCA, et al. Sequencing-based counting and size profiling of plasma Epstein-Barr virus DNA enhance population screening of nasopharyngeal carcinoma. Proc Natl Acad Sci USA. 2018;115(22):E5115-E5124.

10. Chen YP, Chan ATC, Le QT, et al. Nasopharyngeal carcinoma. Lancet. 2019;394(10192):64-80.

11. Au KH, Ngan RKC, Ng AWY, et al. Treatment outcomes of nasopharyngeal carcinoma in modern era after intensity modulated radiotherapy (IMRT) in Hong Kong: A report of 3328 patients (HKNPCSG 1301 study). Oral Oncol. 2018; 77:16-21.

12. Leong YH, Soon YY, Lee KM, et al. Long-term outcomes after reirradiation in nasopharyngeal carcinoma with intensitymodulated radiotherapy: A meta-analysis. Head Neck. 2018;40(3):622-631.

13. Qiu WZ, Huang PY, Shi JL, et al. Neoadjuvant chemotherapy plus intensity-modulated radiotherapy versus concurrent chemoradiotherapy plus adjuvant chemotherapy for the treatment of locoregionally advanced nasopharyngeal carcinoma: a retrospective controlled study. Chin J Cancer. 2016;35:2.

14. Steeg PS. Targeting metastasis. Nat Rev Cancer. 2016;16 (4):201-218.

15. Qu W, Li S, Zhang M, et al. Pattern and prognosis of distant metastases in nasopharyngeal carcinoma: a large-population retrospective analysis. Cancer Med. 2020;9:6147-6158.

16. Chinese Journal of Cancer. The 150 most important questions in cancer research and clinical oncology series: questions 6-14: Edited by Chinese Journal of Cancer. Chin J Cancer. 2017; 36(1):33.

17. Langley RR, Fidler IJ. The seed and soil hypothesis revisited the role of tumor-stroma interactions in metastasis to different organs. Int J Cancer. 2011;128(11):2527-2535. 
18. Blanco MA, Kang Y. Signaling pathways in breast cancer metastasis - novel insights from functional genomics. Breast Cancer Res: BCR. 2011;13(2):206.

19. Khan I, Steeg PS. Metastasis suppressors: functional pathways. Lab Invest. 2018;98(2):198-210.

20. Mei Y, Yang JP, Qian CN. For robust big data analyses: a collection of 150 important pro-metastatic genes. Chin J Cancer. 2017;36(1):16.

21. Qian CN, Mei Y, Zhang J. Cancer metastasis: issues and challenges. Chin J Cancer. 2017;36(1):38.

22. Suhail Y, Cain MP, Vanaja K, et al. Systems biology of cancer metastasis. Cell Syst. 2019;9(2):109-127.

23. Cully M. Tumour microenvironment: Fibroblast subtype provides niche for cancer stem cells. Nat Rev Cancer. 2018;18(3):136.

24. Zhuyan J, Chen M, Zhu T, et al. Critical steps to tumor metastasis: alterations of tumor microenvironment and extracellular matrix in the formation of pre-metastatic and metastatic niche. Cell Biosci. 2020;10:89.

25. Qian CN, Berghuis B, Tsarfaty G, et al. Preparing the "soil": the primary tumor induces vasculature reorganization in the sentinel lymph node before the arrival of metastatic cancer cells. Cancer Res. 2006;66(21):10365-10376.

26. Song LB, Yan J, Jian SW, et al. Molecular mechanisms of tumorgenesis and metastasis in nasopharyngeal carcinoma cell sublines. Ai Zheng/Chin J Cancer. 2002;21(2):158-162.

27. Bao YN, Cao X, Luo DH, et al. Urokinase-type plasminogen activator receptor signaling is critical in nasopharyngeal carcinoma cell growth and metastasis. Cell Cycle. 2014;13(12):1958-1969.

28. Chen WH, Cai MY, Zhang JX, et al. FMNL1 mediates nasopharyngeal carcinoma cell aggressiveness by epigenetically upregulating MTA1. Oncogene. 2018;37(48):6243-6258.

29. Hong X, Liu N, Liang Y, et al.. Circular RNA CRIM1 functions as a ceRNA to promote nasopharyngeal carcinoma metastasis and docetaxel chemoresistance through upregulating FOXQ1. Mol Cancer. 2020;19(1):33.

30. Li GP, Wang H, Lai YK, et al. Proteomic profiling between CNE-2 and its strongly metastatic subclone S-18 and functional characterization of HSP27 in metastasis of nasopharyngeal carcinoma. Proteomics. 2011;11(14):2911-2920.

31. Li XJ, Peng LX, Shao JY, et al. As an independent unfavorable prognostic factor, IL-8 promotes metastasis of nasopharyngeal carcinoma through induction of epithelial-mesenchymal transition and activation of AKT signaling. Carcinogenesis. 2012; 33(7):1302-1309.

32. Lin X, Bi Z, Hu Q, et al. TSPAN8 serves as a prognostic marker involving Akt/MAPK pathway in nasopharyngeal carcinoma. Ann Trans Med. 2019;7(18):470.

33. Meng DF, Sun R, Liu GY, et al. S100A14 suppresses metastasis of nasopharyngeal carcinoma by inhibition of NF-kB signaling through degradation of IRAK1. Oncogene. 2020;39(30):5307-5322.

34. Meng DF, Xie P, Peng LX, et al. CDC42-interacting protein 4 promotes metastasis of nasopharyngeal carcinoma by mediating invadopodia formation and activating EGFR signaling. J Exp Clin Cancer Res. 2017;36(1):21.

35. Qiang YY, Li CZ, Sun R, et al. Along with its favorable prognostic role, CLCA2 inhibits growth and metastasis of nasopharyngeal carcinoma cells via inhibition of FAK/ERK signaling. J Exp Clin Cancer Res. 2018;37(1):34.

36. Sang Y, Chen MY, Luo D, et al. TEL2 suppresses metastasis by down-regulating SERPINE1 in nasopharyngeal carcinoma. Oncotarget. 2015;6(30):29240-29253.
37. Zheng LS, Yang JP, Cao Y, et al. SPINK6 promotes metastasis of nasopharyngeal carcinoma via binding and activation of epithelial growth factor receptor. Cancer Res. 2017;77(2): 579-589.

38. Price JE. Spontaneous and experimental metastasis models: nude mice. Methods Mol Biol. 2014;1070:223-233.

39. Li XJ, Ong CK, Cao Y, et al. Serglycin is a theranostic target in nasopharyngeal carcinoma that promotes metastasis. Cancer Res. 2011;71(8):3162-3172.

40. Shanmugaratnam K. Histological typing of nasopharyngeal carcinoma. IARC Sci Publ. 1978;20:3-12.

41. Cheung F, Chan O, Ng WT, et al. The prognostic value of histological typing in nasopharyngeal carcinoma. Oral Oncol. 2012;48(5):429-433.

42. Wang HY, Chang YL, To KF, et al. A new prognostic histopathologic classification of nasopharyngeal carcinoma. Chin J Cancer. 2016;35:41.

43. Wang HY, Sun BY, Zhu ZH, et al. Eight-signature classifier for prediction of nasopharyngeal [corrected] carcinoma survival. J Clin Oncol. 2011;29(34):4516-4525.

44. Ager A. High endothelial venules and other blood vessels: critical regulators of lymphoid organ development and function. Front Immunol. 2017;8:45.

45. Qian CN, Resau JH, Teh BT. Prospects for vasculature reorganization in sentinel lymph nodes. Cell Cycle. 2007;6(5): 514-517.

46. Qin L, Bromberg-White JL, Qian CN. Opportunities and challenges in tumor angiogenesis research: back and forth between bench and bed. Adv Cancer Res. 2012;113:191-239.

47. Brown M, Assen FP, Leithner A, et al. Lymph node blood vessels provide exit routes for metastatic tumor cell dissemination in mice. Science. 2018;359(6382):1408-1411.

48. Tang F, Tie Y, Hong W, et al. Targeting myeloid-derived suppressor cells for premetastatic niche disruption after tumor resection. Ann Surg Oncol. 2021;28(7):4030-4048.

49. Groth $\mathrm{C}, \mathrm{Hu} \mathrm{X}$, Weber R, et al. Immunosuppression mediated by myeloid-derived suppressor cells (MDSCs) during tumour progression. Br J Cancer. 2019;120(1):16-25.

50. Li ZL, Ye SB, OuYang LY, et al. COX-2 promotes metastasis in nasopharyngeal carcinoma by mediating interactions between cancer cells and myeloid-derived suppressor cells. Oncoimmunology. 2015;4(11):e1044712.

51. Heerboth S, Housman G, Leary M, et al. EMT and tumor metastasis. Clin Trans Med. 2015;4:6.

52. Pastushenko I, Blanpain C. EMT transition states during tumor progression and metastasis. Trends Cell Biol. 2019; 29(3):212-226.

53. Santamaria PG, Moreno-Bueno G, Portillo F, et al. EMT: Present and future in clinical oncology. Mol Oncol. 2017;11(7): 718-738.

54. Ribatti D, Tamma R, Annese T. Epithelial-mesenchymal transition in cancer: a historical overview. Transl Oncol. 2020;13(6): 100773 .

55. Qi XK, Han HQ, Zhang HJ, et al. OVOL2 links stemness and metastasis via fine-tuning epithelial-mesenchymal transition in nasopharyngeal carcinoma. Theranostics. 2018;8(8):2202-2216.

56. Wang MH, Sun R, Zhou XM, et al. Epithelial cell adhesion molecule overexpression regulates epithelial-mesenchymal transition, stemness and metastasis of nasopharyngeal carcinoma cells via the PTEN/AKT/mTOR pathway. Cell Death Dis. 2018;9(1):2. 
57. Zou Y, Yang R, Huang ML, et al. NOTCH2 negatively regulates metastasis and epithelial-Mesenchymal transition via TRAF6/AKT in nasopharyngeal carcinoma. J Exp Clin Cancer Res. 2019;38(1):456.

58. Williams ED, Gao D. Controversies around epithelial-mesenchymal plasticity in cancer metastasis. Nat Rev Cancer. 2019; 19(12):716-732.

59. Lieberman PM. Epstein-Barr virus turns 50. Science. 2014;343 (6177):1323-1325.

60. Zhang H, Li Y, Wang HB, et al. Ephrin receptor A2 is an epithelial cell receptor for Epstein-Barr virus entry. Nat Microbiol. 2018;3(2):1-8.

61. Hau PM, Lung HL, Wu M, et al. Targeting Epstein-Barr virus in nasopharyngeal carcinoma. Front Oncol. 2020;10:600.

62. Elgui de Oliveira D, Muller-Coan BG, Pagano JS. Viral carcinogenesis beyond malignant transformation: EBV in the progression of human cancers. Trends Microbiol. 2016;24(8): 649-664.

63. Takada K. Role of EBER and BARF1 in nasopharyngeal carcinoma (NPC) tumorigenesis. Semin Cancer Biol. 2012; 22(2):162-165.

64. Chirayil R, Kincaid RP, Dahlke C, et al. Identification of virusencoded microRNAs in divergent Papillomaviruses. PLoS Pathog. 2018;14(7) e1007156.

65. Klinke O, Feederle R, Delecluse HJ. Genetics of Epstein-Barr virus microRNAs. Semin Cancer Biol. 2014;26:52-59.

66. Guan S, Wei J, Huang L, et al. Chemotherapy and chemoresistance in nasopharyngeal carcinoma. Eur J Med Chem. 2020;207:112758.

67. Xiang T, Lin YX, Ma W, et al. Vasculogenic mimicry formation in EBV-associated epithelial malignancies. Nat Commun. 2018;9(1):5009.

68. Zhao CX, Zhu W, Ba ZQ, et al. The regulatory network of nasopharyngeal carcinoma metastasis with a focus on EBV, lncRNAs and miRNAs. Am J Cancer Res. 2018;8(11):21852209.

69. Connolly SA, Jackson JO, Jardetzky TS, et al. Fusing structure and function: a structural view of the herpesvirus entry machinery. Nat Rev Microbiol. 2011;9(5):369-381.

70. Wang HB, Zhang H, Zhang JP, et al. Neuropilin 1 is an entry factor that promotes EBV infection of nasopharyngeal epithelial cells. Nat Commun. 2015;6:6240.

71. Zhang HJ, Tian J, Qi XK, et al. Epstein-Barr virus activates F-box protein FBXO2 to limit viral infectivity by targeting glycoprotein B for degradation. PLoS Pathog. 2018;14(7): e1007208.

72. Duan Y, Li Z, Cheng S, et al. Nasopharyngeal carcinoma progression is mediated by EBER-triggered inflammation via the RIG-I pathway. Cancer Lett. 2015;361(1):67-74.

73. Xu M, Zhang WL, Zhu Q, et al. Genome-wide profiling of Epstein-Barr virus integration by targeted sequencing in Epstein-Barr virus associated malignancies. Theranostics. 2019;9(4):1115-1124.

74. Huang T, Yin L, Wu J, et al. TNFAIP3 inhibits migration and invasion in nasopharyngeal carcinoma by suppressing epithelial mesenchymal transition. Neoplasma. 2017;64(3):389-394.

75. Wang $\mathrm{H}$, Jiang Z, Na M, et al. PARK2 negatively regulates the metastasis and epithelial-mesenchymal transition of glioblastoma cells via ZEB1. Oncology Lett. 2017;14(3):2933-2939.

76. Li S, Dai X, Gong K, et al. PA28alpha/beta promote breast cancer cell invasion and metastasis via down-regulation of CDK15. Front Oncol. 2019;9:1283.
77. Wasil LR, Shair KH. Epstein-Barr virus LMP1 induces focal adhesions and epithelial cell migration through effects on integrin-alpha5 and N-cadherin. Oncogenesis. 2015;4:e171.

78. Wei J, Zhang J, Si Y, et al. Blockage of LMP1-modulated storeoperated $\mathrm{Ca}(2+)$ entry reduces metastatic potential in nasopharyngeal carcinoma cell. Cancer Lett. 2015;360(2):234-244.

79. Luo X, Hong L, Cheng C, et al. DNMT1 mediates metabolic reprogramming induced by Epstein-Barr virus latent membrane protein 1 and reversed by grifolin in nasopharyngeal carcinoma. Cell Death Dis. 2018;9(6):619.

80. Liu X, Li Y, Peng S, et al. Epstein-Barr virus encoded latent membrane protein 1 suppresses necroptosis through targeting RIPK1/3 ubiquitination. Cell Death Dis. 2018;9(2):53.

81. Yang CF, Yang GD, Huang TJ, et al. EB-virus latent membrane protein 1 potentiates the stemness of nasopharyngeal carcinoma via preferential activation of PI3K/AKT pathway by a positive feedback loop. Oncogene. 2016;35(26):3419-3431.

82. Lo AK, Liu Y, Wang XH, et al. Alterations of biologic properties and gene expression in nasopharyngeal epithelial cells by the Epstein-Barr virus-encoded latent membrane protein 1. Lab Invest. 2003;83(5):697-709.

83. Li YY, Chung GT, Lui VW, et al. Exome and genome sequencing of nasopharynx cancer identifies NF-kappaB pathway activating mutations. Nat Commun. 2017;8:14121.

84. Zuo LL, Zhang J, Liu LZ, et al. Cadherin 6 is activated by Epstein-Barr virus LMP1 to mediate EMT and metastasis as an interplay node of multiple pathways in nasopharyngeal carcinoma. Oncogenesis. 2017;6(12):402.

85. Horikawa T, Yang J, Kondo S, et al. Twist and epithelialmesenchymal transition are induced by the EBV oncoprotein latent membrane protein 1 and are associated with metastatic nasopharyngeal carcinoma. Cancer Res. 2007;67(5):1970-1978.

86. Dawson CW, Port RJ, Young LS. The role of the EBV-encoded latent membrane proteins LMP1 and LMP2 in the pathogenesis of nasopharyngeal carcinoma (NPC). Semin Cancer Biol. 2012;22(2):144-153.

87. Zhou X, Matskova L, Rathje LS, et al. SYK interaction with ITGbeta4 suppressed by Epstein-Barr virus LMP2A modulates migration and invasion of nasopharyngeal carcinoma cells. Oncogene. 2015;34(34):4491-4499.

88. Richardo T, Prattapong P, Ngernsombat C, et al. Epstein-Barr Virus mediated signaling in nasopharyngeal carcinoma carcinogenesis. Cancers. 2020;12(9):2441.

89. Meyer-Hoffert U, Wu Z, Kantyka T, et al. Isolation of SPINK6 in human skin: selective inhibitor of kallikrein-related peptidases. J Biol Chem. 2010;285(42):32174-32181.

90. Li XJ, Qian CN. Serglycin in human cancers. Chin J Cancer. 2011;30(9):585-589.

91. Chu Q, Huang H, Huang T, et al. Extracellular serglycin upregulates the CD44 receptor in an autocrine manner to maintain self-renewal in nasopharyngeal carcinoma cells by reciprocally activating the MAPK/beta-catenin axis. Cell Death Dis. 2016;7(11):e2456.

92. Waugh DJ, Wilson C. The interleukin-8 pathway in cancer. Clin Cancer Res. 2008;14(21):6735-6741.

93. Wong TS, Chan WS, Li CH, et al. Curcumin alters the migratory phenotype of nasopharyngeal carcinoma cells through upregulation of E-cadherin. Anticancer Res. 2010;30(7):2851-2856.

94. Zheng Z, Pan J, Chu B, et al. Downregulation and abnormal expression of E-cadherin and beta-catenin in nasopharyngeal carcinoma: close association with advanced disease stage and lymph node metastasis. Human Pathol. 1999;30(4):458-466. 
95. Zhang RL, Peng LX, Yang JP, et al. IL-8 suppresses E-cadherin expression in nasopharyngeal carcinoma cells by enhancing E-cadherin promoter DNA methylation. Int J Oncol. 2016; 48(1):207-214.

96. Zhou Y, Kipps TJ, Zhang S. Wnt5a signaling in normal and cancer stem cells. Stem Cells Int. 2017;2017:5295286.

97. Qin L, Yin YT, Zheng FJ, et al. WNT5A promotes stemness characteristics in nasopharyngeal carcinoma cells leading to metastasis and tumorigenesis. Oncotarget. 2015;6(12):1023910252.

98. Yang J, Lv X, Chen J, et al. CCL2-CCR2 axis promotes metastasis of nasopharyngeal carcinoma by activating ERK1/2MMP2/9 pathway. Oncotarget. 2016;7(13):15632-15647.

99. Chen LC, Chen CC, Liang Y, et al. A novel role for TNFAIP2: its correlation with invasion and metastasis in nasopharyngeal carcinoma. Modern Pathol. 2011;24(2):175-184.

100. Sun W, Liu DB, Li WW, et al. Interleukin-6 promotes the migration and invasion of nasopharyngeal carcinoma cell lines and upregulates the expression of MMP-2 and MMP-9. Int J Oncol. 2014;44(5):1551-1560.

101. Wang L, Ma R, Kang Z, et al. Effect of IL-17A on the migration and invasion of NPC cells and related mechanisms. PLoS One. 2014;9(9):e108060.

102. Chen S, Youhong T, Tan Y, et al. EGFR-PKM2 signaling promotes the metastatic potential of nasopharyngeal carcinoma through induction of FOSL1 and ANTXR2. Carcinogenesis. 2020;41(6):863-864.

103. Kjoller L, Hall A. RAC mediates cytoskeletal rearrangements and increased cell motility induced by urokinase-type plasminogen activator receptor binding to vitronectin. J Cell Biol. 2001;152(6):1145-1157.

104. Elliott BE, Meens JA, SenGupta SK, et al. The membrane cytoskeletal crosslinker ezrin is required for metastasis of breast carcinoma cells. Breast Cancer Res. 2005;7(3):R365-R373.

105. Tang Y, Sun X, Yu S, et al. Inhibition of Ezrin suppresses cell migration and invasion in human nasopharyngeal carcinoma. Oncol Lett. 2019;18(1):553-560.

106. Fan C, Qu H, Xiong F, et al. CircARHGAP12 promotes nasopharyngeal carcinoma migration and invasion via ezrinmediated cytoskeletal remodeling. Cancer Lett. 2021;496: $41-56$.

107. Fan C, Tu C, Qi P, et al. GPC6 promotes cell proliferation, migration, and invasion in nasopharyngeal carcinoma. J Cancer. 2019;10(17):3926-3932.

108. Ke L, Xiang Y, Guo X, et al. c-Src activation promotes nasopharyngeal carcinoma metastasis by inducing the epithelial-mesenchymal transition via PI3K/Akt signaling pathway: a new and promising target for NPC. Oncotarget. 2016;7(19): 28340-28355.

109. Zhu JF, Huang W, Yi HM, et al. Annexin A1-suppressed autophagy promotes nasopharyngeal carcinoma cell invasion and metastasis by PI3K/AKT signaling activation. Cell Death Dis. 2018;9(12):1154.

110. Chen CH, Shiu LY, Su LJ, et al. FLJ10540 is associated with tumor progression in nasopharyngeal carcinomas and contributes to nasopharyngeal cell proliferation, and metastasis via osteopontin/CD44 pathway. J Transl Med. 2012;10:93.

111. Peng LX, Wang MD, Xie P, et al. LACTB promotes metastasis of nasopharyngeal carcinoma via activation of ERBB3/EGFRERK signaling resulting in unfavorable patient survival. Cancer Lett. 2021;498:165-177.
112. Sang Y, Cheng C, Zeng YX, et al. Snail promotes metastasis of nasopharyngeal carcinoma partly by down-regulating TEL2. Cancer Commun. 2018;38(1):58.

113. Lian Y, Xiong F, Yang L, et al. Long noncoding RNA AFAP1-AS1 acts as a competing endogenous RNA of miR$423-5 \mathrm{p}$ to facilitate nasopharyngeal carcinoma metastasis through regulating the Rho/Rac pathway. J Exp Clin Cancer Res. 2018;37(1):253.

114. Zheng ZQ, Li ZX, Zhou GQ, et al. Long noncoding RNA FAM225A promotes nasopharyngeal carcinoma tumorigenesis and metastasis by acting as ceRNA to sponge miR-590-3p/ miR-1275 and upregulate ITGB3. Cancer Res. 2019;79 (18):4612-4626.

115. He SW, Xu C, Li YQ, et al. AR-induced long non-coding RNA LINC01503 facilitates proliferation and metastasis via the SFPQ-FOSL1 axis in nasopharyngeal carcinoma. Oncogene. 2020;39(34):5616-5632.

116. He H, Liao X, Yang Q, et al. MicroRNA-494-3p promotes cell growth, migration, and invasion of nasopharyngeal carcinoma by targeting Sox7. Technol Cancer Res Treat. 2018;17: 1533033818809993.

117. Zhao Y, Hong XH, Li K, et al. ZNF582 hypermethylation promotes metastasis of nasopharyngeal carcinoma by regulating the transcription of adhesion molecules Nectin-3 and NRXN3. Cancer Commun. 2020;40(12):721-737.

118. Zhang Y, Sun R, Liu B, et al. TLR3 activation inhibits nasopharyngeal carcinoma metastasis via downregulation of chemokine receptor CXCR4. Cancer Biol Ther. 2009;8(19): 1826-1830.

119. Yuan J, Chen L, Xiao J, et al. SHROOM2 inhibits tumor metastasis through RhoA-ROCK pathway-dependent and independent mechanisms in nasopharyngeal carcinoma. Cell Death Dis. 2019;10(2):58.

120. Chen J, Jiang C, Fu L, et al. CHL1 suppresses tumor growth and metastasis in nasopharyngeal carcinoma by repressing PI3K/AKT signaling pathway via interaction with Integrin $\beta 1$ and Merlin. Int J Biol Sci. 2019;15(9):1802-1815.

121. Chai AW, Cheung AK, Dai W, et al. Metastasis-suppressing NID2, an epigenetically-silenced gene, in the pathogenesis of nasopharyngeal carcinoma and esophageal squamous cell carcinoma. Oncotarget. 2016;7(48):78859-78871.

122. Zhang W, Guo Q, Liu G, et al. NKILA represses nasopharyngeal carcinoma carcinogenesis and metastasis by NF-kappaB pathway inhibition. PLoS Genet. 2019;15(8): e1008325.

123. Wang JG, Tang WP, Liao MC, et al. MiR-99a suppresses cell invasion and metastasis in nasopharyngeal carcinoma through targeting HOXA1. OncoTargets Ther. 2017;10:753-761.

124. Wei F, Wu Y, Tang L, et al. BPIFB1 (LPLUNC1) inhibits migration and invasion of nasopharyngeal carcinoma by interacting with VTN and VIM. Br J Cancer. 2018;118(2): 233-247.

125. Liu W, Cai T, Li L, et al. MiR-200a regulates nasopharyngeal carcinoma cell migration and invasion by targeting MYH10. J Cancer. 2020;11(10):3052-3060.

126. $\mathrm{Hu} \mathrm{W}$, Yao W, Li H, et al. MiR-30e-5p inhibits the migration and invasion of nasopharyngeal carcinoma via regulating the expression of MTA1. Biosci Rep. 2020;40(5):BSR20194309.

127. Tang XR, Wen X, He QM, et al. MicroRNA-101 inhibits invasion and angiogenesis through targeting ITGA3 and its systemic delivery inhibits lung metastasis in nasopharyngeal carcinoma. Cell Death Dis. 2017;8(1):e2566. 
128. Liu N, Jiang N, Guo R, et al. MiR-451 inhibits cell growth and invasion by targeting MIF and is associated with survival in nasopharyngeal carcinoma. Mol Cancer. 2013;12 (1): 123 .

129. Lo KW, Cheung ST, Leung SF, et al. Hypermethylation of the p16 gene in nasopharyngeal carcinoma. Cancer Res. 1996;56 (12):2721-2725.

130. Chan AS, To KF, Lo KW, et al. High frequency of chromosome $3 p$ deletion in histologically normal nasopharyngeal epithelia from southern Chinese. Cancer Res. 2000;60 (19):5365-5370.

131. Lo KW, Teo PM, Hui AB, et al. High resolution allelotype of microdissected primary nasopharyngeal carcinoma. Cancer Res. 2000;60(13):3348-3353.

132. Tang XR, Li YQ, Liang SB, et al. Development and validation of a gene expression-based signature to predict distant metastasis in locoregionally advanced nasopharyngeal carcinoma: a retrospective, multicentre, cohort study. Lancet Oncol. 2018;19(3):382-393.

133. Wang YQ, Zhang Y, Jiang W, et al. Development and validation of an immune checkpoint-based signature to predict prognosis in nasopharyngeal carcinoma using computational pathology analysis. J Immunother Cancer. 2019;7(1):298.

134. Or YY, Chung GT, To KF, et al. Identification of a novel 12p13.3 amplicon in nasopharyngeal carcinoma. J Pathol. 2010;220(1):97-107.

135. Hui AB, Or YY, Takano H, et al. Array-based comparative genomic hybridization analysis identified cyclin D1 as a target oncogene at $11 \mathrm{q} 13.3$ in nasopharyngeal carcinoma. Cancer Res. 2005;65(18):8125-8133.

136. Man CH, Wei-Man Lun S, Wai-Ying Hui J, et al. Inhibition of NOTCH3 signalling significantly enhances sensitivity to cisplatin in EBV-associated nasopharyngeal carcinoma. J Pathol. 2012;226(3):471-481.

Cite this article as: Guo L-L, Wang H-Y, Zheng L-S, Wang M-D, Cao Y, Li Y, Liu Z-J, Peng L-X, Huang B-J, Shao J-Y, and Qian C-N. Metastasis of nasopharyngeal carcinoma: What we know and do not know. Visualized Cancer Medicine. 2021; 2, 4. 